\title{
A note on lifting of Carnot groups
}

\section{Andrea Bonfiglioli and Francesco Uguzzoni}

\begin{abstract}
We prove that every homogeneous Carnot group can be lifted to a free homogeneous Carnot group. Though following the ideas of Rothschild and Stein, we give simple and self-contained arguments, providing a constructive proof, as shown in the examples.
\end{abstract}

\section{Introduction and main results}

The analysis of partial differential operators of the form $\mathcal{L}=\sum_{j \leq m} X_{j}^{2}$, where the $X_{j}$ 's are smooth vector fields on $\mathbb{R}^{N}$, started with Hörmander's paper [15], in which the celebrated maximum rank condition was introduced in relation to the study of the hypoellipticity of $\mathcal{L}$. Afterwards, Rothschild and Stein [18] obtained sharp regularity results for $\mathcal{L}$ by using analysis on nilpotent Lie groups. Harmonic analysis on stratified groups was furthermore systematically developed by Folland [8] and by Folland and Stein [10]. A crucial step in [18] is the construction of vector fields $\widetilde{X}_{1}, \ldots, \widetilde{X}_{m}$ on a manifold larger than $\mathbb{R}^{N}$ which lift the $X_{j}$ 's and which can be locally approximated by left-invariant vector fields on a stratified group.

In recent years, the study of stratified groups (also known as Carnot groups) and their related sub-Laplacians has experienced an increasing development in several different directions of research. Since sub-Laplacians can be viewed as suitable local approximations of general Hörmander sum of squares $\mathcal{L}$, a prominent role in this rising interest is undoubtedly played by the lifting result of Rothschild and Stein.

Different proofs of the lifting procedure have then been provided by other authors (see Hörmander-Melin [16], Folland [9], Goodman [11]) who felt the need to give direct and geometric proofs of this result. In [9], for example,

2000 Mathematics Subject Classification: Primary: 35H20, 43A80; Secondary: 22E60. Keywords: Lifting of vector fields, Carnot groups, fundamental solutions, free groups. 
it is considered the case when $X_{1}, \ldots, X_{m}$ already generate a nilpotent Lie algebra with homogeneous properties.

The aim of our paper is to deal with a case similar to this last one. Indeed, we treat the case when the $X_{j}$ 's generate the Lie algebra of a Carnot group $\mathbb{G}$ and we prove that $\mathbb{G}$ can be directly lifted to a free group $\widetilde{\mathbb{G}}$ which preserves the homogeneous structure of $\mathbb{G}$, besides being itself a homogeneous Carnot group. We shall explain below our main motivation for this precise study. Here, we would like to remark that, even if we follow the idea in [18], our main result (see Theorem 1.1 below) is not explicitly given in none of the papers quoted above. Our scope is also to provide a simple proof, the most direct and self-contained as possible. We investigate homogeneous Carnot groups and the lifting process to free homogeneous ones, from the point of view of PDE's and our arguments are mainly addressed to non specialists in Lie group theory (to this end, the Appendix provides a few background material). The lifting theorem we are concerned with can be summarized as follows.

Theorem 1.1 Let $\mathbb{G}$ be a homogeneous Carnot group on $\mathbb{R}^{N}$. Then, there exists a free homogeneous Carnot group $\widetilde{\mathbb{G}}$ on $\mathbb{R}^{H}$ (with $H \geq N$ ) such that, denoting by $\pi: \mathbb{R}^{H} \rightarrow \mathbb{R}^{N}$ the projection on the first $N$ coordinates (up to a permutation of the coordinates of $\mathbb{R}^{H}$ ), we have

$$
\widetilde{X}_{i}(u \circ \pi)=\left(X_{i} u\right) \circ \pi, \quad \forall u \in C^{\infty}\left(\mathbb{R}^{N}\right),
$$

where $\sum_{i=1}^{m} X_{i}^{2}$ and $\sum_{i=1}^{m} \widetilde{X}_{i}^{2}$ are the canonical sub-Laplacians $\Delta_{\mathbb{G}}$ and $\Delta_{\widetilde{\mathbb{G}}}$, respectively. Moreover $\pi: \widetilde{\mathbb{G}} \rightarrow \mathbb{G}$ is a Lie group morphism.

We refer to Theorem 2.4 for a more detailed statement and to the end of this section for the notation and definitions. We now would like to stress that a lifting result such as Theorem 1.1 (preserving the well-behaved properties of homogeneous Carnot groups) is a crucial step in a wider project which we now briefly recall. Indeed, we are interested in applying analysis on Carnot groups to the study of non-linear subelliptic PDE's arising in the geometric theory of several complex variables such as the Levi-curvature equation, which has achieved rising concern in the last few years (see $[19,17,6,5]$ ). The starting point of this programme is to study the fundamental solution of linear parabolic-type operators in non-divergence form

$$
\mathcal{H}=\sum_{i, j} a_{i, j}(x) X_{i} X_{j}-\partial_{t},
$$

being $\left\{X_{i}\right\}_{i}$ a stratified system of Hörmander vector fields and $a_{i, j}$ Hölder continuous functions. In the forthcoming paper [3], we construct the fundamental solution for $\mathcal{H}$ via the Levi-parametrix method: this is made possible 
by means of the uniform Gaussian estimates established in [2] for the fundamental solutions of the frozen operators. We explicitly remark that in [2] these uniform estimates are derived by making a crucial use of our Theorem 1.1, as we hereafter precise.

Indeed, consider the frozen operators $\mathcal{H}_{A}=\sum_{j=1}^{m} a_{i, j} X_{i} X_{j}-\partial_{t}$, where $A=\left(a_{i, j}\right)$ is a positive-definite matrix belonging to a suitable class of ellipticity. Let $\mathbb{G}$ be the Carnot group related to the $X_{j}$ 's and let $\widetilde{\mathbb{G}}$ be the free Carnot group lifting $\mathbb{G}$ as in Theorem 1.1. The family of heat operators $\left\{\mathcal{H}_{A}\right\}$ is then lifted to a corresponding family $\left\{\widetilde{\mathcal{H}}_{A}\right\}$. Since $\widetilde{\mathbb{G}}$ is free, the operators $\widetilde{\mathcal{H}}_{A}$ are all diffeomorphically equivalent to the canonical one related to the identity matrix: this is proved by the authors in [4] (we highlight that this fact is not true in general, if the group is not free). The related fundamental solutions $\widetilde{\Gamma}_{A}$ are hence all diffeomorphic to the one related to the identity matrix, whence they satisfy the uniform Gaussian estimates in [2, Theorem 2.7]. Finally, the fundamental solutions $\Gamma_{A}$ of $\mathcal{H}_{A}$ are explicitly represented by integrating $\widetilde{\Gamma}_{A}$ with respect to the added variables. As a straightforward consequence, the uniform Gaussian estimates can be obtained for the fundamental solutions $\Gamma_{A}$ on the general non-free group $\mathbb{G}$.

In this paper, we also give a direct example of application of Theorem 1.1 to PDE's. Indeed, in Section 4 we write an explicit formula for the fundamental solutions for all the sub-Laplacians on Carnot groups of step two. This formula is given in terms only of the fundamental solution for the canonical sub-Laplacian on a fixed free Carnot group. The latter fundamental solution can be written in a somewhat explicit form by means of a result by Beals, Gaveau and Greiner [1].

We now introduce a few basic notation and definitions about Carnot groups. Let $\circ$ be an assigned Lie group law on $\mathbb{R}^{N}$. We suppose $\mathbb{R}^{N}$ is endowed with a homogeneous structure by a given family of Lie group automorphisms $\left\{\delta_{\lambda}\right\}_{\lambda>0}$ (called dilations) of the form

$$
\delta_{\lambda}(x)=\delta_{\lambda}\left(x^{(1)}, \ldots, x^{(r)}\right)=\left(\lambda x^{(1)}, \ldots, \lambda^{r} x^{(r)}\right) .
$$

Here $x^{(i)} \in \mathbb{R}^{N_{i}}$ for $i=1, \ldots, r$ and $N_{1}+\cdots+N_{r}=N$. We denote by $\mathfrak{g}$ the Lie algebra of $\left(\mathbb{R}^{N}, \circ\right)$. For $i=1, \ldots, N_{1}$, let $X_{i}$ be the vector field in $\mathfrak{g}$ that agrees at the origin with $\partial / \partial x_{i}^{(1)}$. We make the following assumption: the Lie algebra generated by $X_{1}, \ldots, X_{N_{1}}$ is the whole $\mathfrak{g}$. With the above hypotheses, we call $\mathbb{G}=\left(\mathbb{R}^{N}, \circ, \delta_{\lambda}\right)$ a homogeneous Carnot group (this is not the classical definition, but it is an equivalent one, see Proposition 5.3 and Theorem 5.6 in the Appendix). We also say that $\mathbb{G}$ is of step $r$ and has $m:=$ $N_{1}$ generators. We denote by $Q=\sum_{j=1}^{r} j N_{j}$ the homogeneous dimension of $\mathbb{G}$. The canonical sub-Laplacian on $\mathbb{G}$ is the second order differential 
operator $\Delta_{\mathbb{G}}=\sum_{i=1}^{m} X_{i}^{2}$. If $Y_{1}, \ldots, Y_{m}$ is any basis for $\operatorname{span}\left\{X_{1}, \ldots, X_{m}\right\}$, the second order differential operator $\mathcal{L}=\sum_{i=1}^{m} Y_{i}^{2}$ will be called a subLaplacian on $\mathbb{G}$. We explicitly remark that $\mathcal{L}$ is hypoelliptic since $Y_{1}, \ldots, Y_{m}$ Lie-generate $\mathfrak{g}$ and hence they satisfy Hörmander's condition

$$
\operatorname{rank}\left(\operatorname{Lie}\left\{Y_{1}, \ldots, Y_{m}\right\}(x)\right)=N, \quad \forall x \in \mathbb{R}^{N} .
$$

We now recall the definition of $\mathfrak{f}_{m, r}$, the free nilpotent Lie algebra of step $r$ with $m(\geq 2)$ generators $x_{1}, \ldots, x_{m}$. By definition, $\mathfrak{f}_{m, r}$ is the unique (up to isomorphism) nilpotent Lie algebra of step $r$ generated by $m$ of its elements $x_{1}, \ldots, x_{m}$, such that for every nilpotent Lie algebra $\mathfrak{n}$ of step $r$ and for every map $\varphi$ from $\left\{x_{1}, \ldots, x_{m}\right\}$ to $\mathfrak{n}$, there exists a (unique) Lie algebra morphism $\widetilde{\varphi}$ from $\mathfrak{f}_{m, r}$ to $\mathfrak{n}$ extending $\varphi$. The construction of such a Lie algebra $\mathfrak{f}_{m, r}$ is classical (see e.g., $[20,21]$ ). We say that the Carnot group $\mathbb{G}$ is a free Carnot group if its Lie algebra $\mathfrak{g}$ is isomorphic to $\mathfrak{f}_{m, r}$, for some $m$ and $r . \mathbb{R}^{N}$ equipped with the ordinary abelian structure is an example of free Carnot group. The Heisenberg group $\mathbb{H}^{1}$ is also a free Carnot group, while $\mathbb{H}^{n}$ is not free, for any $n \geq 2$, as can be seen by a dimensional argument. We refer the reader to [14] for the construction of a basis for $\mathfrak{f}_{m, r}$ (see also [12]).

The paper is organized as follows. Section 2 contains the proof of our main result Theorem 2.4: the proof describes in details how to realize the lifting process. Section 3 provides an explicit example of our lifting, illustrating the constructive proof of the previous section. In Section 4, we prove our explicit formula for the fundamental solutions on step two Carnot groups. Finally, the Appendix collects a few results on Carnot groups. We warn the reader that known basic properties of Carnot groups will be used throughout Section 2 and 3, without mention. Some of these properties are recalled in the Appendix, where we also prove that our operative definition of homogeneous Carnot group is equivalent to the classical one.

\section{Lifting to free Carnot groups}

For the rest of this section, $(\mathbb{G}, \circ)$ will be a (fixed) homogeneous Carnot group on $\mathbb{R}^{N}$ with dilations $\delta_{\lambda}\left(x^{(1)}, \ldots, x^{(r)}\right)=\left(\lambda x^{(1)}, \ldots, \lambda^{r} x^{(r)}\right), x^{(i)} \in \mathbb{R}^{N_{i}}$, and we set for brevity $m=N_{1}$. Moreover, $\mathcal{Z}=\left(Z_{1}, \cdots, Z_{N}\right)$ will denote the Jacobian basis of the Lie algebra $\mathfrak{g}$ of $\mathbb{G}$, i.e., $Z_{i}$ is the vector field in $\mathfrak{g}$ agreeing at the origin with $\partial / \partial x_{i}$. We explicitly recall that $\mathfrak{g}$ is a $N$-dimensional nilpotent Lie algebra of step $r$ generated by $Z_{1}, \ldots, Z_{m}$. If $Z_{j}^{(i)} \in \mathfrak{g}$ agrees at the origin with $\partial / \partial x_{j}^{(i)}$, then it is easy to see that $Z_{j}^{(i)}$ is $\delta_{\lambda^{-}}$ homogeneous of degree $i$. Finally, let $\mathfrak{f}_{m, r}$ be the free nilpotent Lie algebra of step $r$, with $m$ generators $F_{1}, \ldots, F_{m}$. By the definition of $\mathfrak{f}_{m, r}$, there exists 
a unique Lie algebra morphism $\Pi$ from $\mathfrak{f}_{m, r}$ to $\mathfrak{g}$ such that $\Pi\left(F_{i}\right)=Z_{i}(i=$ $1, \ldots, m)$. Clearly, $\Pi$ is surjective, whence $\operatorname{dim} \mathfrak{f}_{m, r}=\operatorname{dim} \operatorname{ker}(\Pi)+\operatorname{dim} \mathfrak{g}$. We set $H=\operatorname{dim} \mathfrak{f}_{m, r}$. The following result will be of relevance in the sequel.

Proposition 2.1 There exists a basis $\mathcal{F}=\left(F_{1}, \ldots, F_{H}\right)$ for $\mathfrak{f}_{m, r}$ such that

$$
\Pi\left(F_{j}\right)=Z_{j}, \quad j=1, \ldots, N, \quad \Pi\left(F_{j}\right)=0, \quad j=N+1, \ldots, H,
$$

and each $F_{j}(j=1, \ldots, H)$ is a homogeneous Lie polynomial in $F_{1}, \ldots, F_{m}$.

Proof. Let $\alpha_{j}$ denote the $\delta_{\lambda}$-homogeneity degree of $Z_{j}$. In particular $\alpha_{1}=$ $\ldots=\alpha_{m}=1$. For a multi-index $I=\left(i_{1}, \ldots, i_{k}\right)$ with $i_{1}, \ldots, i_{k} \in\{1, \ldots, m\}$, we set $|I|=k$ (the height of $I$ ) and define $Z_{I}:=\left[Z_{i_{1}},\left[Z_{i_{2}} \ldots\left[Z_{i_{k-1}}, Z_{i_{k}}\right] \ldots\right]\right.$. Then, by simple homogeneity arguments, we have

$$
Z_{j}=\sum_{I \in \mathcal{I}_{j}} c_{I}^{(j)} Z_{I}, \quad c_{I}^{(j)} \in \mathbb{R},
$$

where $\mathcal{I}_{j}$ is a set of multi-indices all with height $\alpha_{j}$. If we analogously set

$$
F_{I}:=\left[F_{i_{1}},\left[F_{i_{2}} \ldots\left[F_{i_{k-1}}, F_{i_{k}}\right] \ldots\right]\right],
$$

the first $N$ elements of the basis $\mathcal{F}$ can be chosen as

$$
F_{j}=\sum_{I \in \mathcal{I}_{j}} c_{I}^{(j)} F_{I}, \quad j=1, \ldots, N .
$$

Indeed $\Pi\left(F_{j}\right)=Z_{j}$ and $F_{1}, \ldots, F_{N}$ are linearly independent homogeneous Lie polynomials in the generators $F_{1}, \ldots, F_{m}$. Let now $\widehat{F}_{N+1}, \ldots, \widehat{F}_{H}$ be a basis for $\operatorname{ker}(\Pi)$. We can write

$$
\widehat{F}_{j}=\sum_{I \in \mathcal{A}_{j}} q_{I}^{(j)} F_{I}, \quad j=N+1, \ldots, H,
$$

for a certain set of multi-indices $\mathcal{A}_{j}$ and scalars $q_{I}^{(j)}$ 's. For every $k=1, \ldots, r$, we set $\mathcal{A}_{j}^{(k)}:=\left\{I \in \mathcal{A}_{j}:|I|=k\right\}$. Then

$$
\widehat{F}_{j}=\sum_{I \in \mathcal{A}_{j}^{(1)}} q_{I}^{(j)} F_{I}+\cdots+\sum_{I \in \mathcal{A}_{j}^{(r)}} q_{I}^{(j)} F_{I}=: \widehat{F}_{j}^{(1)}+\cdots+\widehat{F}_{j}^{(r)} .
$$

The system of vectors $\widehat{\mathcal{F}}:=\left\{\widehat{F}_{j}^{(k)}: j=N+1, \ldots, H, k=1, \ldots, r\right\}$ has the following properties: each of its vectors is a homogeneous Lie polynomial in the generators $F_{1}, \ldots, F_{m}$; the system spans $\operatorname{ker}(\Pi)$; for every $j=N+$ $1, \ldots, H$ and $k=1, \ldots, r$, we have $\widehat{F}_{j}^{(k)} \in \operatorname{ker}(\Pi)$. The last assertion can be proved by simple homogeneity arguments. Finally, the lemma is proved by extracting from the system of vectors $\widehat{\mathcal{F}}$ a basis $F_{N+1}, \ldots, F_{H}$ for $\operatorname{ker}(\Pi)$. 
In the sequel, we shall consider some abstract finite-dimensional algebras; we hence introduce a useful notation, which will allow us to restrict to deal with ordinary $\mathbb{R}^{n}$ spaces. Let $\mathfrak{h}$ be a finite-dimensional nilpotent real Lie algebra. It is possible to prove that $\mathfrak{h}$ is equipped with a Lie group structure by the so-called Campbell-Hausdorff composition law, defined by (5.4) in the Appendix (see, for example, Corollary 5.5). For $X, Y \in \mathfrak{h}$, the first few terms in the sum (5.4) (which is finite since $\mathfrak{h}$ is nilpotent) are given by

$$
X \diamond Y=X+Y+\frac{1}{2}[X, Y]+\frac{1}{12}[X,[X, Y]]-\frac{1}{12}[Y,[X, Y]]+\cdots
$$

We explicitly remark that $\diamond$ is defined in a universal way (independent of $\mathfrak{h}$ ) as a Lie polynomial in $X$ and $Y$. We now fix a basis $\mathcal{E}=\left(E_{1}, \ldots, E_{N}\right)$ for $\mathfrak{h}(N:=\operatorname{dim} \mathfrak{h})$ and we identify $\mathfrak{h}$ with $\mathbb{R}^{N}$ via the map $\pi_{\mathcal{E}}: \mathfrak{h} \rightarrow \mathbb{R}^{N}$, $\sum_{i=1}^{N} \xi_{i} E_{i} \mapsto\left(\xi_{1}, \ldots, \xi_{N}\right)$. The group law $\diamond$ is then turned into a group law $\diamond_{\mathcal{E}}$ on $\mathbb{R}^{N}$ in the natural way:

$$
a \diamond_{\mathcal{E}} b:=\pi_{\mathcal{E}}\left(\pi_{\mathcal{E}}^{-1}(a) \diamond \pi_{\mathcal{E}}^{-1}(b)\right), \quad a, b \in \mathbb{R}^{N} .
$$

The Lie groups $\left(\mathbb{R}^{N}, \diamond_{\mathcal{E}}\right)$ and $(\mathfrak{h}, \diamond)$ are clearly isomorphic via $\pi_{\mathcal{E}}$. We stress that the Lie group morphism $\pi_{\mathcal{E}}$ is also a linear map.

With the above notation, we now consider the Lie groups $\left(\mathbb{R}^{N}, \diamond_{\mathcal{Z}}\right)$ and $\left(\mathbb{R}^{H}, \diamond_{\mathcal{F}}\right)$, where $\mathcal{Z}$ is the Jacobian basis of $\mathfrak{g}$, whereas $\mathcal{F}$ is the basis of $\mathfrak{f}_{m, r}$ introduced in Proposition 2.1. Let $\mathfrak{r}^{N}$ and $\mathfrak{r}^{H}$ denote their Lie algebras, respectively. First of all, we remark that the map

$$
\pi:=\pi_{\mathcal{Z}} \circ \Pi \circ \pi_{\mathcal{F}}^{-1}:\left(\mathbb{R}^{H}, \diamond_{\mathcal{F}}\right) \rightarrow\left(\mathbb{R}^{N}, \diamond_{\mathcal{Z}}\right)
$$

is a surjective Lie group morphism coinciding with the usual projection of $\mathbb{R}^{H}$ onto $\mathbb{R}^{N}$. Indeed, if $\xi \in \mathbb{R}^{H}$ we have (by means of $(2.1)$ )

$$
\pi(\xi)=\pi_{\mathcal{Z}}\left(\sum_{i=1}^{H} \xi_{i} \Pi\left(F_{i}\right)\right)=\pi_{\mathcal{Z}}\left(\sum_{i=1}^{N} \xi_{i} Z_{i}\right)=\left(\xi_{1}, \ldots, \xi_{N}\right) .
$$

We now turn to the Lie algebras: since $\pi$ is a Lie group morphism, then its differential $\mathrm{d} \pi: \mathfrak{r}^{H} \rightarrow \mathfrak{r}^{N}$ is a Lie algebra morphism with the following property (see the Appendix, Theorem 5.2)

$$
(\mathrm{d} \pi(E))_{\pi(\xi)}=\mathrm{d} \pi\left(E_{\xi}\right), \quad \forall E \in \mathfrak{r}^{H}, \quad \forall \xi \in \mathbb{R}^{H} .
$$

Roughly speaking, the differential of a Lie group morphism which coincides with a projection gives a lifting of vector fields. More precisely, we have the following lemma. 
Lemma 2.2 With the above notations, if $E \in \mathfrak{r}^{H}$ then $E$ is a lifting of $\mathrm{d} \pi(E)$ in the following sense: if $f \in C^{\infty}\left(\mathbb{R}^{H}\right)$ depends only on $\xi_{1}, \ldots, \xi_{N}$ i.e., $f\left(\xi_{1}, \ldots, \xi_{H}\right)=g\left(\xi_{1}, \ldots, \xi_{N}\right)$ for every $\xi \in \mathbb{R}^{H}\left(\right.$ with $g \in C^{\infty}\left(\mathbb{R}^{N}\right)$ ), then we have

$$
(E f)\left(\xi_{1}, \ldots, \xi_{H}\right)=(\mathrm{d} \pi(E) g)\left(\xi_{1}, \ldots, \xi_{N}\right), \quad \forall \xi \in \mathbb{R}^{H} .
$$

Proof. Let $g \in C^{\infty}\left(\mathbb{R}^{N}\right)$ and $f \in C^{\infty}\left(\mathbb{R}^{H}\right)$ be such that $f=g \circ \pi$. We have to prove $E_{\xi}(f)=(\mathrm{d} \pi(E))_{\pi(\xi)}(g)$, for every $\xi \in \mathbb{R}^{H}$. From (2.2), we immediately obtain $(\mathrm{d} \pi(E))_{\pi(\xi)}(g)=\left(\mathrm{d} \pi\left(E_{\xi}\right)\right)(g)=E_{\xi}(g \circ \pi)=E_{\xi}(f)$. This ends the proof.

The rest of the lifting method consists in transferring this result to the group $\mathbb{G}$, after a suitable definition of the larger group which projects onto $\mathbb{G}$. We refer the reader to the following diagram of Lie group morphisms:

$$
\begin{aligned}
& \left(\mathfrak{f}_{m, r}, \diamond\right) \stackrel{\Pi}{\longrightarrow}(\mathfrak{g}, \diamond) \stackrel{\operatorname{Exp}}{\longleftrightarrow}(\mathbb{G}, \circ) \\
& \pi_{\mathcal{F}} \uparrow \quad \uparrow \pi_{\mathcal{Z}} \\
& \left(\mathbb{G} \times \mathbb{R}^{H-N}, \bullet\right) \stackrel{\Phi}{\longleftrightarrow}\left(\mathbb{R}^{H}, \diamond_{\mathcal{F}}\right) \stackrel{\pi}{\longrightarrow}\left(\mathbb{R}^{N}, \diamond_{\mathcal{Z}}\right)
\end{aligned}
$$

We recall that $\left(\mathbb{R}^{N}, \diamond_{\mathcal{Z}}\right)$ is isomorphic to $(\mathfrak{g}, \diamond)$ via $\pi_{\mathcal{Z}}$. On the other hand, the exponential map $\operatorname{Exp}:(\mathfrak{g}, \diamond) \rightarrow(\mathbb{G}, \circ)$ is a Lie group isomorphism. As a consequence, the map $\Psi:=\operatorname{Exp} \circ \pi_{\mathcal{Z}}^{-1}$ is a Lie group isomorphism from $\left(\mathbb{R}^{N}, \diamond_{\mathcal{Z}}\right)$ to $(\mathbb{G}, \circ)$.

We then look for a suitable group structure on $\mathbb{G} \times \mathbb{R}^{H-N}$ and a Lie group isomorphism

$$
\Phi:\left(\mathbb{R}^{H}, \diamond_{\mathcal{F}}\right) \rightarrow\left(\mathbb{G} \times \mathbb{R}^{H-N}, \bullet\right)
$$

such that $\vartheta:=\Psi \circ \pi \circ \Phi^{-1}$ is the projection of $\mathbb{G} \times \mathbb{R}^{H-N}$ onto $\mathbb{G}$. To this end, we set

$$
\Phi\left(\xi_{1}, \ldots, \xi_{H}\right):=\left(\Psi\left(\xi_{1}, \ldots, \xi_{N}\right), \xi_{N+1}, \ldots, \xi_{H}\right) .
$$

Clearly, $\Phi$ is a diffeomorphism of class $C^{\infty}$. We then define on $\mathbb{G} \times \mathbb{R}^{H-N}$ the composition law induced by $\Phi$

$$
\left(g_{1}, a_{1}\right) \bullet\left(g_{2}, a_{2}\right):=\Phi\left(\Phi^{-1}\left(g_{1}, a_{1}\right) \diamond_{\mathcal{F}} \Phi^{-1}\left(g_{2}, a_{2}\right)\right),
$$

so that $\Phi$ becomes a Lie group isomorphism between $\left(\mathbb{R}^{H}, \diamond_{\mathcal{F}}\right)$ and $(\mathbb{G} \times$ $\left.\mathbb{R}^{H-N}, \bullet\right)$. Finally, the Lie group morphism

$$
\vartheta:=\Psi \circ \pi \circ \Phi^{-1}:\left(\mathbb{G} \times \mathbb{R}^{H-N}, \bullet\right) \rightarrow(\mathbb{G}, \circ)
$$

is, by construction, the natural projection. Indeed,

$$
\begin{aligned}
\vartheta\left(x_{1}, \ldots, x_{H}\right) & =(\Psi \circ \pi)\left(\Psi^{-1}\left(x_{1}, \ldots, x_{N}\right), x_{N+1}, \ldots, x_{H}\right) \\
& =\Psi\left(\Psi^{-1}\left(x_{1}, \ldots, x_{N}\right)\right)=\left(x_{1}, \ldots, x_{N}\right) .
\end{aligned}
$$


We denote by $\mathfrak{g} \mathfrak{r}^{H-N}$ the Lie algebra of $\left(\mathbb{G} \times \mathbb{R}^{H-N}, \bullet\right)$. The proof of the following result is simply a restatement of the proof of Lemma 2.2.

Lemma 2.3 If $W \in \mathfrak{g r}^{H-N}$, then $W$ is a lifting of $\mathrm{d} \vartheta(W)$ in the following sense: if $f \in C^{\infty}\left(\mathbb{G} \times \mathbb{R}^{H-N}\right)$ depends only on $x_{1}, \ldots, x_{N}$, i.e., $f\left(x_{1}, \ldots, x_{H}\right)=g\left(x_{1}, \ldots, x_{N}\right)$ for every $x \in \mathbb{G} \times \mathbb{R}^{H-N}\left(\right.$ with $\left.g \in C^{\infty}(\mathbb{G})\right)$, then we have

$$
(W f)\left(x_{1}, \ldots, x_{H}\right)=(\mathrm{d} \vartheta(W) g)\left(x_{1}, \ldots, x_{N}\right), \quad \forall x \in \mathbb{G} \times \mathbb{R}^{H-N} .
$$

We claim that the first $N$ vector fields of the Jacobian basis $W_{1}, \ldots, W_{H}$ for $\mathfrak{g} \mathfrak{r}^{H-N}$ lift orderly the Jacobian basis $Z_{1}, \ldots, Z_{N}$ for $\mathfrak{g}$. Indeed, by Lemma 2.3, it suffices to show that $\mathrm{d} \vartheta\left(W_{k}\right)=Z_{k}$ for every $k=1, \ldots, N$. On the other hand, for all $f \in C^{\infty}(\mathbb{G})$ we have

$$
\left(\mathrm{d} \vartheta\left(W_{k}\right)\right)_{0}(f)=\left(W_{k}\right)_{0}(f \circ \vartheta)=\left(\partial_{x_{k}} f\right)(0)=\left(Z_{k}\right)_{0} f .
$$

We are now in the position to prove the main result of this section.

Theorem 2.4 (Lifting) Let $\mathbb{G}$ be a homogeneous Carnot group on $\mathbb{R}^{N}$ of step $r$ and $m\left(=N_{1}\right)$ generators. Then, there exists a free homogeneous Carnot group $\widetilde{\mathbb{G}}$ on $\mathbb{R}^{H}\left(H=\operatorname{dim} \mathfrak{f}_{m, r}\right)$ with the properties (i) and (ii) stated below.

We fix the following notations:

$$
\begin{aligned}
& \delta_{\lambda}(x)=\delta_{\lambda}\left(x^{(1)}, x^{(2)}, \ldots, x^{(r)}\right)=\left(\lambda x^{(1)}, \lambda^{2} x^{(2)}, \ldots, \lambda^{r} x^{(r)}\right), \\
& \widetilde{\delta}_{\lambda}(\widetilde{x})=\widetilde{\delta}_{\lambda}\left(\widetilde{x}^{(1)}, \widetilde{x}^{(2)}, \ldots, \widetilde{x}^{(r)}\right)=\left(\lambda \widetilde{x}^{(1)}, \lambda^{2} \widetilde{x}^{(2)}, \ldots, \lambda^{r} \widetilde{x}^{(r)}\right)
\end{aligned}
$$

denote the dilations on $\mathbb{G}$ and $\widetilde{\mathbb{G}}$, respectively $\left(x^{(i)} \in \mathbb{R}^{N_{i}} i=1, \ldots, r\right.$, $\left.N_{1}+\cdots+N_{r}=N ; \widetilde{x}^{(i)} \in \mathbb{R}^{\widetilde{N}_{i}} i=1, \ldots, r, \widetilde{N}_{1}+\cdots+\widetilde{N}_{r}=H\right) ; Z_{j}^{(i)}$ $\left(i \leq r, j \leq N_{i}\right.$ ) denote the Jacobian basis of the Lie algebra $\mathfrak{g}$ of $\mathbb{G}$ and $\widetilde{Z}_{j}^{(i)}$ $\left(i \leq r, j \leq \widetilde{N}_{i}\right)$ denote the Jacobian basis of the Lie algebra $\widetilde{\mathfrak{g}}$ of $\widetilde{\mathbb{G}}$.

(i) $\widetilde{\mathbb{G}}$ has step $r$ and $m$ generators and its Lie algebra is isomorphic to $\mathfrak{f}_{m, r}$.

(ii) For a certain $i_{0} \in\{1, \ldots, r\}$ we have

$$
\widetilde{N}_{i}=N_{i} \quad\left(i=1, \ldots, i_{0}\right) \quad \text { and } \quad \tilde{N}_{i}>N_{i} \quad\left(i=i_{0}+1, \ldots, r\right) ;
$$

moreover, if $\varrho^{(i)}: \mathbb{R}^{\widetilde{N}_{i}} \rightarrow \mathbb{R}^{N_{i}}$ denotes the projection on the first $N_{i}$ coordinates and $\varrho: \mathbb{R}^{H} \rightarrow \mathbb{R}^{N}$ is defined by $\varrho(\widetilde{x})=\left(\varrho^{(1)}\left(\widetilde{x}^{(1)}\right), \ldots, \varrho^{(r)}\left(\widetilde{x}^{(r)}\right)\right)$, then

$$
\widetilde{Z}_{j}^{(i)}(u \circ \varrho)=\left(Z_{j}^{(i)} u\right) \circ \varrho, \quad \forall u \in C^{\infty}\left(\mathbb{R}^{N}\right), \quad i \leq r, j \leq N_{i},
$$

i.e., $\widetilde{Z}_{j}^{(i)}$ lifts $Z_{j}^{(i)}$. Moreover, $\varrho$ is a Lie group morphism. 
Proof. Let $\left(\mathbb{G} \times \mathbb{R}^{H-N}, \bullet\right)$ be the Lie group on $\mathbb{R}^{H}$ previously defined. We show that $\widetilde{G}$ can be constructed from $\mathbb{G} \times \mathbb{R}^{H-N}$ by a permutation of the coordinates. Let $\mathcal{F}=\left(F_{1}, \ldots, F_{H}\right)$ be the basis for $\mathfrak{f}_{m, r}$ as in Proposition 2.1. Then $F_{j}$ is a homogeneous Lie polynomial of degree $\alpha_{j}$ in the generators $F_{1}, \ldots, F_{m}$. We stress that, by Proposition $2.1, \alpha_{j}$ is also the $\delta_{\lambda}$-homogeneity degree of $Z_{j}$, for $j=1, \ldots, N$, i.e., the dilation $\delta_{\lambda}$ on $\mathbb{R}^{N}$ can be written as $\delta_{\lambda}\left(x_{1}, \ldots, x_{N}\right)=\left(\lambda^{\alpha_{1}} x_{1}, \ldots, \lambda^{\alpha_{N}} x_{N}\right)$. We also observe that only $F_{1}, \ldots, F_{m}$ have degree 1 . We define dilations on $\mathfrak{f}_{m, r}$ as follows:

$$
\widehat{\delta}_{\lambda}\left(\sum_{i=1}^{H} \xi_{i} F_{i}\right):=\sum_{i=1}^{H} \lambda^{\alpha_{i}} \xi_{i} F_{i} .
$$

First we prove that $\widehat{\delta}_{\lambda}$ is a Lie algebra automorphism of $\mathfrak{f}_{m, r}$. Indeed, for all $i, j \in\{1, \ldots, H\}$

$$
\widehat{\delta}_{\lambda}\left(\left[F_{i}, F_{j}\right]\right)=\lambda^{\alpha_{i}+\alpha_{j}}\left[F_{i}, F_{j}\right]=\left[\lambda^{\alpha_{i}} F_{i}, \lambda^{\alpha_{j}} F_{j}\right]=\left[\widehat{\delta}_{\lambda}\left(F_{i}\right), \widehat{\delta}_{\lambda}\left(F_{j}\right)\right] .
$$

The first equality holds since $\left[F_{i}, F_{j}\right]$ is a homogeneous Lie polynomial in $F_{1}, \ldots, F_{m}$ of degree $\alpha_{i}+\alpha_{j}$. Then, $\widehat{\delta}_{\lambda}$ is also a Lie group automorphism of $\left(\mathfrak{f}_{m, r}, \diamond\right)$, by Remark 5.5. As a consequence, $\delta_{\lambda}^{*}:=\pi_{\mathcal{F}} \circ \widehat{\delta}_{\lambda} \circ \pi_{\mathcal{F}}^{-1}$ is a Lie group automorphism of $\left(\mathbb{R}^{H}, \diamond_{\mathcal{F}}\right)$. Analogously, $\widetilde{\delta}_{\lambda}:=\Phi \circ \delta_{\lambda}^{*} \circ \Phi^{-1}$ is a Lie group automorphism of $\left(\mathbb{G} \times \mathbb{R}^{H-N}, \bullet\right)$. If $x \in \mathbb{G} \times \mathbb{R}^{H-N}$, we have

$$
\begin{aligned}
\widetilde{\delta}_{\lambda}(x) & =\left(\left(\Psi \circ \delta_{\lambda} \circ \Psi^{-1}\right)\left(x_{1}, \ldots, x_{N}\right), \lambda^{\alpha_{N+1}} x_{N+1}, \ldots, \lambda^{\alpha_{H}} x_{H}\right) \\
& =\left(\delta_{\lambda}\left(x_{1}, \ldots, x_{N}\right), \lambda^{\alpha_{N+1}} x_{N+1}, \ldots, \lambda^{\alpha_{H}} x_{H}\right) .
\end{aligned}
$$

Indeed, as we recall in the Appendix, $\Psi$ commutes with the dilations of $\mathbb{G}$.

We then reorder the coordinates of $\mathbb{G} \times \mathbb{R}^{H-N}$ in the following way. Let $(x, y) \in \mathbb{G} \times \mathbb{R}^{H-N}$, where $x=\left(x^{(1)}, \ldots, x^{(r)}\right) \in \mathbb{G}$ and $y=\left(y_{N+1}, \ldots, y_{H}\right) \in$ $\mathbb{R}^{H-N}$. We can suppose that the coordinates of $y$ are ordered in such a way that $\alpha_{N+1} \leq \cdots \leq \alpha_{H}$. Setting $i_{0}:=\alpha_{N+1}-1$, we can write $y=\left(y^{\left(i_{0}+1\right)}, \ldots, y^{(r)}\right)$, where to each coordinate $y_{l}$ of $y^{(k)}\left(i_{0}+1 \leq k \leq r\right)$ corresponds a degree of homogeneity $\alpha_{l}$ equal to $k$. We now set

$$
P: \mathbb{G} \times \mathbb{R}^{H-N} \rightarrow \mathbb{R}^{H}, \quad(x, y) \mapsto\left(x^{(1)} ; \ldots ; x^{\left(i_{0}\right)} ; x^{\left(i_{0}+1\right)}, y^{\left(i_{0}+1\right)} ; \ldots ; x^{(r)}, y^{(r)}\right) .
$$

We define $\widetilde{\mathbb{G}}$ as $\mathbb{R}^{H}$ with the group structure naturally induced by $P$ from $\left(\mathbb{G} \times \mathbb{R}^{H-N}, \bullet\right)$. With respect to the coordinates of $\widetilde{\mathbb{G}}, \widetilde{\delta}_{\lambda}$ assumes the usual form

$$
\left(\lambda x^{(1)} ; \ldots ; \lambda^{i_{0}} x^{\left(i_{0}\right)} ; \lambda^{i_{0}+1} x^{\left(i_{0}+1\right)}, \lambda^{i_{0}+1} y^{\left(i_{0}+1\right)} ; \ldots ; \lambda^{r} x^{(r)}, \lambda^{r} y^{(r)}\right) .
$$

We now set $\varrho:=\vartheta \circ P^{-1}$. It is then easy to recognize that (2.3) follows from Lemma 2.3. 
In order to complete the proof, we have to show that $\widetilde{\mathbb{G}}$ is a homogeneous Carnot group with Lie algebra $\widetilde{\mathfrak{g}}$ isomorphic to $\mathfrak{f}_{m, r}$. To this purpose, let $\left(\mathbb{G}_{m, r}, *\right)$ be a free homogeneous Carnot group on $\mathbb{R}^{H}$ whose Lie algebra is isomorphic to $\mathfrak{f}_{m, r}$ : the existence of such a group can be easily derived by the Third Fundamental Theorem of Lie and by our Theorem 5.6. It is then non-restrictive to consider $\mathfrak{f}_{m, r}$ to be the Lie algebra of $\mathbb{G}_{m, r}$ itself. If we denote by $\operatorname{Exp}_{*}$ the exponential map from $\left(\mathfrak{f}_{m, r}, \diamond\right)$ to $\left(\mathbb{G}_{m, r}, *\right)$, then

$$
\operatorname{Exp}_{*} \circ \pi_{\mathcal{F}}^{-1}:\left(\mathbb{R}^{H}, \diamond_{\mathcal{F}}\right) \rightarrow\left(\mathbb{G}_{m, r}, *\right)
$$

is a Lie group isomorphism. As a consequence, by Theorem 5.2 in the Appendix, $\mathrm{d}\left(\operatorname{Exp}_{*} \circ \pi_{\mathcal{F}}^{-1}\right)$ is a Lie algebra isomorphism from $\mathfrak{r}^{H}$ to $\mathfrak{f}_{m, r}$. Let $E_{1}, \ldots, E_{H}$ be the Jacobian basis for $\mathfrak{r}^{H}$. We now prove that

$$
\mathrm{d}\left(\operatorname{Exp}_{*} \circ \pi_{\mathcal{F}}^{-1}\right)\left(E_{i}\right)=F_{i}, \quad i=1, \ldots, m .
$$

In particular, since $F_{1}, \ldots, F_{m}$ are generators for $\mathfrak{f}_{m, r}$, this will prove that $E_{1}, \ldots, E_{m}$ are generators for $\mathfrak{r}^{H}$. If $f \in C^{\infty}\left(\mathbb{G}_{m, r}\right)$, we have

$$
\begin{aligned}
\left(\mathrm{d}\left(\operatorname{Exp}_{*} \circ \pi_{\mathcal{F}}^{-1}\right)\left(E_{i}\right)\right)_{0}(f) & =\left(E_{i}\right)_{0}\left(f \circ \operatorname{Exp}_{*} \circ \pi_{\mathcal{F}}^{-1}\right) \\
& =\left(\left.\partial_{\xi_{i}}\right|_{\xi=0}\right) f\left(\operatorname{Exp}_{*}\left(\sum_{j=1}^{H} \xi_{j} F_{j}\right)\right) \\
& =\left.\frac{\mathrm{d}}{\mathrm{d} t}\right|_{t=0} f\left(\operatorname{Exp}_{*}\left(t F_{i}\right)\right) \\
& =\left.\frac{\mathrm{d}}{\mathrm{d} t}\right|_{t=0} f\left(\exp _{F_{i}}(t)\right)=\left(F_{i}\right)_{0}(f)
\end{aligned}
$$

Since a left-invariant vector field is determined by its value at the origin, this proves (2.4). An analogous argument shows that

$$
\mathrm{d} \Phi: \mathfrak{r}^{H} \rightarrow \mathfrak{g r}^{H-N}
$$

maps the first $m$ vector fields of the Jacobian basis for $\mathfrak{r}^{H}$ into the first $m$ vector fields of the Jacobian basis for $\mathfrak{g r}^{H-N}$. Finally, since $\widetilde{\mathbb{G}}$ is obtained from $\mathbb{G} \times \mathbb{R}^{H-N}$ by the Lie group isomorphism $P$ (which permutes the coordinates, leaving the first $m$ ones unaltered) we can assert that $\widetilde{\mathbb{G}}$ is a homogeneous Carnot group. Moreover, $\widetilde{\mathfrak{g}}$ is isomorphic to $\mathfrak{f}_{m, r}$ via the Lie algebra isomorphism

$$
\mathrm{d}\left(\operatorname{Exp}_{*} \circ \pi_{\mathcal{F}}^{-1} \circ \Phi^{-1} \circ P^{-1}\right)
$$

This ends the proof.

In Section 3 below, we give an example of the lifting method just described. 


\section{An example of lifting}

We here give an example of a homogeneous Carnot group and we lift it to a free homogeneous Carnot group. We shall follow the notations introduced in Section 2. We consider the homogeneous Carnot group $\mathbb{G}$ on $\mathbb{R}^{5}$ with group law and dilations defined as follows: $\delta_{\lambda}(x)=\left(\lambda x_{1}, \lambda x_{2}, \lambda x_{3}, \lambda x_{4}, \lambda^{2} x_{5}\right)$ and

$$
x \circ y=\left(x_{1}+y_{1}, x_{2}+y_{2}, x_{3}+y_{3}, x_{4}+y_{4}, x_{5}+y_{5}+x_{1} y_{3}+x_{2} y_{4}\right) .
$$

The Jacobian basis $\mathcal{Z}$ for $\mathfrak{g}$ is given by

$$
Z_{1}=\partial_{1}, Z_{2}=\partial_{2}, Z_{3}=\partial_{3}+x_{1} \partial_{5}, Z_{4}=\partial_{4}+x_{2} \partial_{5}, Z_{5}=\partial_{5} .
$$

The Campbell-Hausdorff formula on a Lie algebra nilpotent of step 2 is given by $X \diamond Y=X+Y+\frac{1}{2}[X, Y]$. Hence the group $\left(\mathbb{R}^{5}, \diamond_{\mathcal{Z}}\right)$ has composition law given by:

$$
\begin{aligned}
\xi \diamond_{\mathcal{Z}} \eta=( & \xi_{1}+\eta_{1}, \xi_{2}+\eta_{2}, \xi_{3}+\eta_{3}, \xi_{4}+\eta_{4}, \\
& \left.\xi_{5}+\eta_{5}+\frac{1}{2}\left(\xi_{1} \eta_{3}-\xi_{3} \eta_{1}+\xi_{2} \eta_{4}-\xi_{4} \eta_{2}\right)\right) .
\end{aligned}
$$

With few modifications, we recognize that $\diamond_{\mathcal{Z}}$ is the usual group law on $\mathbb{H}^{2}$, the Heisenberg group on $\mathbb{R}^{5}$. The Jacobian basis $\mathcal{X}$ for $\mathfrak{r}^{5}$ is given by:

$X_{1}=\partial_{1}-\frac{\xi_{3}}{2} \partial_{5}, X_{2}=\partial_{2}-\frac{\xi_{4}}{2} \partial_{5}, X_{3}=\partial_{3}+\frac{\xi_{1}}{2} \partial_{5}, X_{4}=\partial_{4}+\frac{\xi_{2}}{2} \partial_{5}, X_{5}=\partial_{5}$.

We now turn to consider $\mathfrak{f}_{4,2}$. We have $\operatorname{dim} \mathfrak{f}_{4,2}=10$. Let $\Pi: \mathfrak{f}_{4,2} \rightarrow \mathfrak{g}$ be the Lie algebra morphism such that $\Pi\left(F_{i}\right)=Z_{i}$ for every $i=1, \ldots, 4$. The following is a basis $\mathcal{F}$ for $\mathfrak{f}_{4,2}$ as in Proposition 2.1:

$F_{1}, F_{2}, F_{3}, F_{4},\left[F_{1}, F_{3}\right] ;\left[F_{1}, F_{2}\right],\left[F_{1}, F_{4}\right],\left[F_{2}, F_{3}\right],\left[F_{3}, F_{4}\right],\left[F_{2}, F_{4}\right]-\left[F_{1}, F_{3}\right]$.

One can easily recognize that the last 5 vectors in $\mathcal{F}$ form a basis for $\operatorname{ker}(\Pi)$. The Lie group $\left(\mathbb{R}^{10}, \diamond_{\mathcal{F}}\right)$ has composition law given by:

$$
\begin{aligned}
\xi \diamond_{\mathcal{F}} \eta=( & \xi_{1}+\eta_{1}, \xi_{2}+\eta_{2}, \xi_{3}+\eta_{3}, \xi_{4}+\eta_{4}, \\
& \xi_{5}+\eta_{5}+\frac{1}{2}\left(\xi_{1} \eta_{3}-\xi_{3} \eta_{1}+\xi_{2} \eta_{4}-\xi_{4} \eta_{2}\right), \xi_{6}+\eta_{6}+\frac{1}{2}\left(\xi_{1} \eta_{2}-\xi_{2} \eta_{1}\right), \\
& \xi_{7}+\eta_{7}+\frac{1}{2}\left(\xi_{1} \eta_{4}-\xi_{4} \eta_{1}\right), \xi_{8}+\eta_{8}+\frac{1}{2}\left(\xi_{2} \eta_{3}-\xi_{3} \eta_{2}\right), \\
& \left.\xi_{9}+\eta_{9}+\frac{1}{2}\left(\xi_{3} \eta_{4}-\xi_{4} \eta_{3}\right), \xi_{10}+\eta_{10}+\frac{1}{2}\left(\xi_{2} \eta_{4}-\xi_{4} \eta_{2}\right)\right) .
\end{aligned}
$$

The first 5 vector fields of the Jacobian basis for $\mathfrak{r}^{10}$ are then given by:

$$
\begin{aligned}
& E_{1}=\partial_{1}-\frac{\xi_{3}}{2} \partial_{5}-\frac{\xi_{2}}{2} \partial_{6}-\frac{\xi_{4}}{2} \partial_{7}, \quad E_{2}=\partial_{2}-\frac{\xi_{4}}{2} \partial_{5}+\frac{\xi_{1}}{2} \partial_{6}-\frac{\xi_{3}}{2} \partial_{8}-\frac{\xi_{4}}{2} \partial_{10}, \\
& E_{3}=\partial_{3}+\frac{\xi_{1}}{2} \partial_{5}+\frac{\xi_{2}}{2} \partial_{8}-\frac{\xi_{4}}{2} \partial_{9}, \\
& E_{4}=\partial_{4}+\frac{\xi_{2}}{2} \partial_{5}+\frac{\xi_{1}}{2} \partial_{7}+\frac{\xi_{3}}{2} \partial_{9}+\frac{\xi_{2}}{2} \partial_{10}, \\
& E_{5}=\partial_{5} .
\end{aligned}
$$


It is evident that $E_{i}$ lifts $\mathrm{d} \pi\left(E_{i}\right)=X_{i}$, for every $i=1, \ldots, 5$, as stated in Lemma 2.2. By a straightforward calculation of the exponential map from $\mathfrak{g}$ to $\mathbb{G}$, we have

$\Psi=\operatorname{Exp} \circ \pi_{\mathcal{Z}}^{-1}:\left(\mathbb{R}^{5}, \diamond_{\mathcal{Z}}\right) \rightarrow(\mathbb{G}, \circ), \quad \Psi(\xi)=\left(\xi_{1}, \xi_{2}, \xi_{3}, \xi_{4}, \xi_{5}+\frac{1}{2}\left(\xi_{1} \xi_{3}+\xi_{2} \xi_{4}\right)\right)$.

In particular, the Lie group isomorphism $\Phi:\left(\mathbb{R}^{10}, \diamond_{\mathcal{F}}\right) \rightarrow\left(\mathbb{G} \times \mathbb{R}^{5}, \bullet\right)$ is defined by

$$
\Phi(\xi)=\left(\xi_{1}, \xi_{2}, \xi_{3}, \xi_{4}, \xi_{5}+\frac{1}{2}\left(\xi_{1} \xi_{3}+\xi_{2} \xi_{4}\right) ; \quad \xi_{6}, \xi_{7}, \xi_{8}, \xi_{9}, \xi_{10}\right) .
$$

As a consequence, the group law on $\mathbb{G} \times \mathbb{R}^{5}$ is given by:

$$
\begin{aligned}
x \bullet y= & \left(x_{1}+y_{1}, x_{2}+y_{2}, x_{3}+y_{3}, x_{4}+y_{4}, x_{5}+y_{5}+x_{1} y_{3}+x_{2} y_{4},\right. \\
& x_{6}+y_{6}+\frac{1}{2}\left(x_{1} y_{2}-x_{2} y_{1}\right), x_{7}+y_{7}+\frac{1}{2}\left(x_{1} y_{4}-x_{4} y_{1}\right), \\
& x_{8}+y_{8}+\frac{1}{2}\left(x_{2} y_{3}-x_{3} y_{2}\right), x_{9}+y_{9}+\frac{1}{2}\left(x_{3} y_{4}-x_{4} y_{3}\right), \\
& \left.x_{10}+y_{10}+\frac{1}{2}\left(x_{2} y_{4}-x_{4} y_{2}\right)\right) .
\end{aligned}
$$

The first 5 vector fields of the Jacobian basis for $\mathfrak{g}^{5}$ are then given by:

$$
\begin{aligned}
& W_{1}=\partial_{1}-\frac{x_{2}}{2} \partial_{6}-\frac{x_{4}}{2} \partial_{7}, \quad W_{2}=\partial_{2}+\frac{x_{1}}{2} \partial_{6}-\frac{x_{3}}{2} \partial_{8}-\frac{x_{4}}{2} \partial_{10}, \\
& W_{3}=\partial_{3}+x_{1} \partial_{5}+\frac{x_{2}}{2} \partial_{8}-\frac{x_{4}}{2} \partial_{9}, \quad W_{4}=\partial_{4}+x_{2} \partial_{5}+\frac{x_{1}}{2} \partial_{7}+\frac{x_{3}}{2} \partial_{9}+\frac{x_{2}}{2} \partial_{10}, \\
& W_{5}=\partial_{5} .
\end{aligned}
$$

As stated in Lemma $2.3, W_{i}$ lifts $\mathrm{d} \vartheta\left(W_{i}\right)=Z_{i}$, for every $i=1, \ldots, 5$. Finally, in this case the free homogeneous Carnot group $\widetilde{\mathbb{G}}$ which lifts $\mathbb{G}$ has the same group law as $\left(\mathbb{G} \times \mathbb{R}^{5}, \bullet\right)$ since the permutation of the coordinates $P$ can be chosen as the identity map. We remark that the dilations of $\widetilde{\mathbb{G}}$ are

$$
\delta_{\lambda}(x)=\left(\lambda x_{1}, \lambda x_{2}, \lambda x_{3}, \lambda x_{4}, \lambda^{2} x_{5}, \lambda^{2} x_{6}, \lambda^{2} x_{7}, \lambda^{2} x_{8}, \lambda^{2} x_{9}, \lambda^{2} x_{10}\right) .
$$

\section{An example of application to PDE's}

In this Section, we focus on Carnot groups of step two. We characterize the composition law on such groups and we explicitly exhibit their lifting. As a consequence, we derive a direct formula for the fundamental solutions for all the sub-Laplacians on groups of step two. This formula is given in terms only of the fundamental solution for the canonical sub-Laplacian on a fixed free Carnot group. The latter fundamental solution can be written in a somewhat explicit form by means of a result by Beals, Gaveau and Greiner [1].

The result below easily follows from Proposition 5.1 in the Appendix. 
Remark 4.1 The $N$-dimensional homogeneous Carnot groups of step two and $m$ generators are characterized by being $\left(\mathbb{R}^{N}, \circ\right)$ with the following Lie group law $\left(N=m+n, x \in \mathbb{R}^{m}, t \in \mathbb{R}^{n}\right)$

$$
(x, t) \circ(\xi, \tau)=\left(\begin{array}{c}
x_{j}+\xi_{j}, \quad j=1, \ldots, m \\
t_{j}+\tau_{j}+\frac{1}{2}\left\langle x, B^{(j)} \xi\right\rangle, \quad j=1, \ldots, n
\end{array}\right),
$$

where the $B^{(j)}$ 's are $m \times m$ matrices, whose skew-symmetric parts $\frac{1}{2}\left(B^{(j)}-\right.$ $\left(B^{(j)}\right)^{T}$ ) are linearly independent. (Up to a Lie group isomorphism, the matrices $B^{(j)}$ 's can be supposed to be themselves skew-symmetric.)

We fix $\mathbb{G}=\left(\mathbb{R}^{N}, \circ\right)$ a Carnot group of step two as above, i.e., we fix matrices $B^{(1)}, \ldots, B^{(n)}$ as in Remark 4.1. Let $\mathcal{L}=\sum_{j=1}^{m} Y_{j}^{2}$ be a fixed subLaplacian on $\mathbb{G}$. Then, there exists a non-singular $m \times m$ matrix $A=\left(a_{k, j}\right)_{k, j}$ such that $Y_{j}=\sum_{k=1}^{m} a_{k, j} Z_{k}$, where $Z_{1}, \ldots, Z_{m}$ denote the first $m$ vector fields of the Jacobian basis of $\mathfrak{g}$. Our aim here is to write the fundamental solution for $\mathcal{L}$ in terms only of $A, B^{(1)}, \ldots, B^{(n)}$ and the fundamental solution for the canonical sub-Laplacian of the prototype free Carnot group $\left(\mathbb{F}_{m, 2}, \star\right)$ which we describe below. Let $i, j \in\{1, \ldots m\}$ with $i>j$ be fixed and let $S^{(i, j)}$ be the $m \times m$ skew-symmetric matrix whose entries are +1 in the position $(i, j),-1$ in the position $(j, i)$ and 0 elsewhere. Then, we agree to denote by $\left(\mathbb{F}_{m, 2}, \star\right)$ the Carnot group associated to these $m(m-1) / 2$ matrices according to Remark 4.1. Here and in the sequel we shall use the following notation: we denote the point of $\mathbb{F}_{m, 2}$ by $(x, \gamma)$ and we also write the coordinates of $\gamma$ by $\gamma_{i, j}$ where $(i, j)$ varies in the set $\mathcal{I}=\{(i, j) \mid 1 \leq j<$ $i \leq m\}$. Then, the composition law $\star$ is given by

$$
(x, \gamma) \star\left(x^{\prime}, \gamma^{\prime}\right)=\left(\begin{array}{c}
x_{h}+x_{h}^{\prime}, \quad h=1, \ldots, m \\
\gamma_{i, j}+\gamma_{i, j}^{\prime}+\frac{1}{2}\left(x_{i} x_{j}^{\prime}-x_{j} x_{i}^{\prime}\right), \quad(i, j) \in \mathcal{I}
\end{array}\right) .
$$

It is easily proved that the Lie algebra of $\left(\mathbb{F}_{m, 2}, \star\right)$ is (isomorphic to) $\mathfrak{f}_{m, 2}$. Then $\mathbb{F}_{m, 2}$ is a free homogeneous Carnot group of step two on $\mathbb{R}^{m(m+1) / 2}$, with $m$ generators.

Since, by assumption, the skew-symmetric parts of $B^{(1)}, \ldots, B^{(n)}$ are linearly independent, there exists indices $\left(i_{1}, j_{1}\right), \ldots,\left(i_{n}, j_{n}\right) \in \mathcal{I}$ such that the following $n \times n$ matrix

$$
\left(\left(A^{T} \frac{B^{(r)}-\left(B^{(r)}\right)^{T}}{2} A\right)_{i_{s}, j_{s}}\right)_{1 \leq r, s \leq n}
$$

is non-singular. We denote by $K=\left(k_{r, s}\right)_{r, s}$ the inverse of the above matrix. We also define the subset of indices $\mathcal{C}=\mathcal{I} \backslash\left\{\left(i_{1}, j_{1}\right), \ldots,\left(i_{n}, j_{n}\right)\right\}$. With the above notation, we shall also denote the point of $\mathbb{F}_{m, 2}$ by $(x, t, \beta)$, where $x \in \mathbb{R}^{m}, t \in \mathbb{R}^{n}, \beta=\left(\beta_{h, k}\right)_{(h, k) \in \mathcal{C}} \in \mathbb{R}^{m(m-1) / 2-n}$. We are now in the position to state the main result of this section. 
Proposition 4.2 With the above notations, let $\Gamma_{\mathcal{L}}$ and $\Gamma_{m, 2}$ denote respectively the fundamental solutions of $\mathcal{L}$ and of $\Delta_{\mathbb{F}_{m, 2}}$. Then, the following formula holds

$$
\begin{aligned}
\Gamma_{\mathcal{L}}(\mathbf{x}, \mathbf{t})=\frac{|\operatorname{det} K|}{|\operatorname{det} A|} \int_{\beta \in \mathbb{R}^{m(m-1) / 2-n}} \mathrm{~d} \beta & \Gamma_{m, 2}\left(A^{-1} \mathbf{x},\left[\sum _ { s \leq n } k _ { r , s } \left(\mathbf{t}_{s}-\frac{1}{4}\left\langle\mathbf{x}, B^{(s)} \mathbf{x}\right\rangle\right.\right.\right. \\
& \left.\left.\left.-\sum_{(h, k) \in \mathcal{C}} \beta_{h, k}\left(A^{T} \frac{B^{(s)}-\left(B^{(s)}\right)^{T}}{2} A\right)_{h, k}\right)\right]_{r \leq n}, \beta\right) .
\end{aligned}
$$

The above result can be applied in order to manage, with uniformity, families of sub-Laplacians (letting $A$ vary) and families of step two Carnot groups with the same number of generators (letting $B^{(1)}, \ldots, B^{(n)}$ vary). Indeed, an example of application is given in $[2,3]$ where uniform estimates for some families of sub-Laplacians are derived and, as a consequence, the fundamental solutions for non-divergence form operators with Hölder continuous coefficients are constructed.

Remark 4.3 We explicitly remark that a rather explicit formula is given by Beals, Gaveau and Greiner in [1], for the fundamental solution of the canonical sub-Laplacian on any step two Carnot group $\mathbb{G}$ :

$$
\Gamma_{\Delta_{\mathbb{G}}}(x, t)=\int_{\mathbb{R}^{n}} V(\rho) f(x, t, \rho)^{(2-Q) / 2} \mathrm{~d} \rho,
$$

where $f$ is the action associated to a complex Hamiltonian problem and $V$ solves a transport equation. Collecting together this formula in the case of the free group $\mathbb{F}_{m, 2}$ and our Proposition 4.2, we obtain the following formula:

$$
\begin{gathered}
\Gamma_{\mathcal{L}}(\mathbf{x}, \mathbf{t})=\frac{|\operatorname{det} K|}{|\operatorname{det} A|} \int_{\beta \in \mathbb{R}^{m(m-1) / 2-n}} \mathrm{~d} \beta \int_{\rho \in \mathbb{R}^{m(m-1) / 2}} \mathrm{~d} \rho\left\{\operatorname{det}\left(\frac{S(\rho)}{\sinh S(\rho)}\right)\right\}^{1 / 2} \times \\
\times\left\{\frac{1}{2}\left\langle S(\rho) \operatorname{coth} S(\rho) A^{-1} \mathbf{x}, A^{-1} \mathbf{x}\right\rangle-\iota\left(\sum _ { r = 1 } ^ { n } \rho _ { i _ { r } , j _ { r } } \left[\sum _ { s \leq n } k _ { r , s } \left(\mathbf{t}_{s}-\frac{1}{4}\left\langle\mathbf{x}, B^{(s)} \mathbf{x}\right\rangle\right.\right.\right.\right. \\
\left.\left.\left.\left.\quad-\sum_{(h, k) \in \mathcal{C}} \beta_{h, k}\left(A^{T} \frac{B^{(s)}-\left(B^{(s)}\right)^{T}}{2} A\right)_{h, k}\right)\right]+\sum_{i, j \in \mathcal{C}} \rho_{i, j} \beta_{i, j}\right)\right\}^{\left(2-m^{2}\right) / 2}
\end{gathered}
$$

Here, $S(\rho)$ is the matrix $S(\rho)=-\iota \sum_{i, j \in \mathcal{I}} \rho_{i, j} S^{(i, j)}$, where the matrices $S^{(i, j)}$ have been introduced above and $\iota$ is the imaginary unit. As an example, if $m=3, \Gamma_{3,2}$ has been explicitly written in [1, p.322]. Hence, the above 
formula writes

$$
\begin{aligned}
& \Gamma_{\mathcal{L}}(\mathbf{x}, \mathbf{t})=-\frac{\operatorname{Gamma}(7 / 2)}{(2 \pi)^{7 / 2}} \frac{|\operatorname{det} K|}{|\operatorname{det} A|} \int_{\beta \in \mathbb{R}^{3-n}} \mathrm{~d} \beta \int_{\rho \in \mathbb{R}^{3}} \mathrm{~d} \rho \frac{2|\rho|}{\sinh (2|\rho|)} \times \\
& \times\left\{\frac{\left\langle A^{-1} \mathbf{x}, \rho\right\rangle^{2}}{2|\rho|^{2}}+|\rho| \operatorname{coth}(2|\rho|)\left[\left|A^{-1} \mathbf{x}\right|^{2}-\frac{\left\langle A^{-1} \mathbf{x}, \rho\right\rangle^{2}}{|\rho|^{2}}+\right.\right. \\
& \quad-\iota\left(\sum_{r=1}^{n} \rho_{r}\left\{\sum_{s \leq n} k_{r, s}\left(\mathbf{t}_{s}-\frac{1}{4}\left\langle\mathbf{x}, B^{(s)} \mathbf{x}\right\rangle-\sum_{(h, k) \in \mathcal{C}} \beta_{h, k}\left(A^{T} \frac{B^{(s)}-\left(B^{(s)}\right)^{T}}{2} A\right)_{h, k}\right)\right\}+\right. \\
& \left.\left.\left.\quad+\sum_{r=n+1}^{3} \rho_{r} \beta_{r-n}\right)\right]\right\}^{-7 / 2} .
\end{aligned}
$$

Proof of Proposition 4.2. As a first step, we turn the arbitrary subLaplacian $\mathcal{L}$ on $\mathbb{G}$ into the canonical sub-Laplacian on a new group $\overline{\mathbb{G}}$ : this can be done by the isomorphism of Lie groups $\overline{\mathbb{G}} \ni(\bar{x}, \bar{t}) \mapsto(A \bar{x}, \bar{t}) \in \mathbb{G}$ which turns the composition $\circ$ on $\mathbb{G}$ into

$$
(\bar{x}, \bar{t}) \overline{\mathrm{o}}\left(\bar{x}^{\prime}, \bar{t}^{\prime}\right)=\left(\begin{array}{c}
\bar{x}_{j}+\bar{x}_{j}^{\prime}, \quad j=1, \ldots, m, \\
\bar{t}_{j}+\bar{t}_{j}^{\prime}+\frac{1}{2}\left\langle\bar{x}, A^{T} B^{(j)} A \bar{x}^{\prime}\right\rangle, \quad j=1, \ldots, n
\end{array}\right) .
$$

Then, it is easy to see that we have the relation

$$
\Gamma_{\mathcal{L}}(x, t)=|\operatorname{det} A|^{-1} \Gamma_{\Delta_{\bar{G}}}\left(A^{-1} x, t\right) .
$$

As a second step, we turn the composition law $\overline{0}$ into a composition law $\underline{\circ}$ whose associated matrices (according to Remark 4.1) are skew-symmetric. This can be done by identifying $\overline{\mathbb{G}}$ with its Lie algebra via the exponential map. Then, the isomorphism of Lie groups

$$
\underline{\mathbb{G}} \ni(\underline{x}, \underline{t}) \mapsto\left(\underline{x}, \underline{t}_{j}+\frac{1}{4}\left\langle\underline{x}, A^{T} B^{(j)} A \underline{x}\right\rangle\right) \in \overline{\mathbb{G}}
$$

turns the composition $\bar{o}$ on $\overline{\mathbb{G}}$ into

$$
(\underline{x}, \underline{t}) \underline{\circ}\left(\underline{x}^{\prime}, \underline{t}^{\prime}\right)=\left(\begin{array}{c}
\underline{x}_{j}+\underline{x}_{j}^{\prime}, \quad j=1, \ldots, m, \\
\underline{t}_{j}+\underline{t}_{j}^{\prime}+\frac{1}{2}\left\langle\underline{x}, A^{T}\left(\frac{B^{(j)}-\left(B^{(j)}\right)^{T}}{2}\right) A \underline{x}^{\prime}\right\rangle, \quad j=1, \ldots, n
\end{array}\right),
$$

where the associated matrices are skew-symmetric. It is easy to see that we have the relation

$$
\Gamma_{\Delta_{\bar{G}}}(\bar{x}, \bar{t})=\Gamma_{\Delta_{\underline{\mathbb{G}}}}\left(\bar{x}, \bar{t}_{j}-\frac{1}{4}\left\langle\bar{x}, A^{T} B^{(j)} A \bar{x}\right\rangle\right) .
$$


As a third step, we lift $\underline{\mathbb{G}}$ to a free Carnot group $\underline{\mathbb{G}}$ as in Theorem 2.4. In [2, Theorem 2.4] it is proved that the fundamental solution of the canonical sub-Laplacian on $\mathbb{\mathbb { G }}$ can be obtained by integrating the fundamental solution of the canonical sub-Laplacian on $\underline{\widetilde{\mathbb{G}}}$ with respect to the added variables, i.e.,

$$
\Gamma_{\Delta_{\underline{\mathbb{G}}}}(\underline{x}, \underline{t})=\int_{\beta \in \mathbb{R}^{m(m-1) / 2-n}} \Gamma_{\Delta_{\underline{\mathbb{G}}}}(\underline{x}, \underline{t}, \beta) \mathrm{d} \beta .
$$

We need the explicit form of the lifting for an arbitrary group of step two (with associated skew-symmetric matrices). This is given in the following lemma, which can be proved retracing the proof of Theorem 2.4.

Lemma 4.4 Let $\left(\mathbb{R}^{N}\right.$, o) be a Carnot group of step two and $m$ generators as in Remark 4.1, where its associated matrices $B^{(j)}$ 's are linearly independent and skew-symmetric.

Then, $\left(\mathbb{R}^{N}, \circ\right)$ is lifted to the group $\left(\mathbb{R}^{N} \times \mathbb{R}^{m(m-1) / 2-n}, \widetilde{o}\right)$ (according to Theorem 2.4), where ( $x \in \mathbb{R}^{m}, t \in \mathbb{R}^{n}, \beta \in \mathbb{R}^{m(m-1) / 2-n}$ )

$$
(x, t, \beta) \circ\left(x^{\prime}, t^{\prime}, \beta^{\prime}\right)=\left(\begin{array}{c}
x_{j}+x_{j}^{\prime}, \quad j=1, \ldots, m \\
t_{j}+t_{j}^{\prime}+\frac{1}{2}\left\langle x, B^{(j)} x^{\prime}\right\rangle, \quad j=1, \ldots, n \\
\beta_{i, j}+\beta_{i, j}^{\prime}+\frac{1}{2}\left(x_{i} x_{j}^{\prime}-x_{j} x_{i}^{\prime}\right), \quad(i, j) \in \mathcal{C}
\end{array}\right) .
$$

The next step is to explicitly write a Lie group isomorphism between $\underline{\mathbb{G}}$ and $\mathbb{F}_{m, 2}$. This can be obtained by the composition $\operatorname{Exp} \circ \varphi \circ \log =: T$, where Exp is the exponential map on $\mathbb{F}_{m, 2}, \log$ is the logarithmic map on $\underline{\mathbb{G}}$, and $\varphi$ is the unique Lie algebra morphism mapping the first $m$ vector fields of the Jacobian basis related to $\underline{\mathbb{G}}$ into the first $m$ vector fields of the Jacobian basis related to $\mathbb{F}_{m, 2}$. A laborious computation shows that the map $T:(\underline{\mathbb{G}}, \underline{\widetilde{o}}) \rightarrow\left(\mathbb{F}_{m, 2}, \star\right)$, is defined by

$T(x, t, \beta):=\left(x,\left[\sum_{s=1}^{n} k_{r, s}\left(t_{s}-\sum_{(h, k) \in \mathcal{C}} \beta_{h, k}\left(A^{T} \frac{B^{(s)}-\left(B^{(s)}\right)^{T}}{2} A\right)_{h, k}\right)\right]_{r \leq n}, \beta\right)$.

It is easy to see that we have the relation

$$
\Gamma_{\Delta_{\widetilde{\mathbb{G}}}}(\underline{x}, \underline{t}, \beta)=|\operatorname{det} K| \Gamma_{m, 2}(T(\underline{x}, \underline{t}, \beta)) .
$$

Finally, collecting together equations (4.1) to (4.4), we obtain the desired formula in Proposition 4.2. 


\section{Appendix: Basic results on Carnot groups}

The main aim of this section is to compare the definition of homogeneous Carnot group in the introduction, to the one usually given in literature. To this end, we first recall some known results on homogeneous Lie groups.

We say that a Lie group $\mathbb{G}=\left(\mathbb{R}^{N}, \circ\right)$ is homogeneous if it admits a group of automorphisms $\left\{\delta_{\lambda}\right\}_{\lambda>0}$ of the form (1.1). Let $\mathfrak{g}$ denote the Lie algebra of $\mathbb{G}$, i.e., the set of o-left-invariant vector fields on $\mathbb{G}$. If $\tau_{x}$ denotes the left-translation by $x$ on $\mathbb{G}$, then a vector field $X$ belongs to $\mathfrak{g}$ if and only if $X(x)=\mathcal{J}_{\tau_{x}}(0) X(0)$, for every $x \in \mathbb{G}\left(\mathcal{J}_{\tau_{x}}\right.$ denotes the Jacobian matrix of $\left.\tau_{x}\right)$. The map $J: \mathbb{R}^{N} \rightarrow \mathfrak{g}, \eta \mapsto X$ defined by $X(x)=\mathcal{J}_{\tau_{x}}(0) \eta$ is then an isomorphism of vector spaces. As a consequence, any basis for $\mathfrak{g}$ is the image via $J$ of a basis of $\mathbb{R}^{N}$. We call the Jacobian basis of $\mathfrak{g}$ the one resulting from the canonical basis of $\mathbb{R}^{N}$, i.e., the basis of vector fields in $\mathfrak{g}$ agreeing at the origin with the coordinate partial derivatives.

A real function $a(x)$ defined on $\mathbb{G}$ is called $\delta_{\lambda}$-homogeneous of degree $\beta \in \mathbb{R}$ if, for every $x \in \mathbb{G}$ and $\lambda>0$, it holds $a\left(\delta_{\lambda}(x)\right)=\lambda^{\beta} a(x)$. A linear differential operator $X$ is called $\delta_{\lambda}$-homogeneous of degree $\beta \in \mathbb{R}$ if, for every $\varphi \in C^{\infty}(\mathbb{G})$ and $\lambda>0$, it holds $X\left(\varphi \circ \delta_{\lambda}\right)=\lambda^{\beta}(X \varphi) \circ \delta_{\lambda}$. It follows that the only smooth $\delta_{\lambda}$-homogeneous functions of degree $\beta$ are the polynomial functions of the form $\sum_{\gamma} c_{\gamma} x^{\gamma}$, where the sum is carried over the multi-indices $\gamma$ such that $\sum_{i=1}^{r} \sum_{j=1}^{N_{i}} i \gamma_{j}^{(i)}=\beta$. Consequently, a smooth vector field $\delta_{\lambda}$-homogeneous of degree $k \leq r(k \in \mathbb{N})$ has the following form

$$
\sum_{i=k}^{r} \sum_{j=1}^{N_{i}} a_{j}^{(i)}\left(x^{(1)}, \ldots, x^{(i-k)}\right) \cdot\left(\partial / \partial x_{j}^{(i)}\right),
$$

where $a_{j}^{(i)}$ is a $\delta_{\lambda}$-homogeneous polynomial of degree $i-k$. In particular, a smooth vector field $\delta_{\lambda}$-homogeneous of degree $k>r$ is necessarily the null operator.

The following result describes in an "explicit" way the composition law of $\mathbb{G}$ and the Lie algebra $\mathfrak{g}$.

Proposition 5.1 Following the notation in (1.1)

$$
x \circ y=\left((x \circ y)^{(1)}, \ldots,(x \circ y)^{(r)}\right),
$$

we have

$$
(x \circ y)^{(1)}=x^{(1)}+y^{(1)}, \quad(x \circ y)^{(i)}=x^{(i)}+y^{(i)}+Q^{(i)}(x, y), \quad 2 \leq i \leq r,
$$

where

1. $Q^{(i)}$ depends only on $x^{(1)}, \ldots, x^{(i-1)}$ and $y^{(1)}, \ldots, y^{(i-1)}$;

2. the component functions of $Q^{(i)}$ are sums of mixed monomials in $x$ and $y$;

3. $Q^{(i)}\left(\delta_{\lambda} x, \delta_{\lambda} y\right)=\lambda^{i} Q^{(i)}(x, y)$. 
This has many relevant consequences. Indeed, we have

$$
\mathcal{J}_{\tau_{x}}(0)=\left(\begin{array}{cccc}
\mathbb{I}_{N_{1}} & 0 & \cdots & 0 \\
J_{2}^{(1)}(x) & \mathbb{I}_{N_{2}} & \ddots & \vdots \\
\vdots & \ddots & \ddots & 0 \\
J_{r}^{(1)}(x) & \cdots & J_{r}^{(r-1)}(x) & \mathbb{I}_{N_{r}}
\end{array}\right)
$$

where $\mathbb{I}_{n}$ is the $n \times n$ identity matrix, whereas $J_{j}^{(i)}(x)$ is a $N_{j} \times N_{i}$ matrix whose entries are $\delta_{\lambda}$-homogeneous polynomials of degree $j-i$. In particular, if we let $\mathcal{J}_{\tau_{x}}(0)=\left(Z^{(1)}(x) \cdots Z^{(r)}(x)\right.$ ) (where $Z^{(i)}(x)$ is a $N \times N_{i}$ matrix), then the column vectors of $Z^{(i)}(x)$ (the Jacobian basis for $\mathfrak{g}$ ) define $\delta_{\lambda^{-}}$ homogeneous vector fields of degree $i$. Consequently, the adjoint operator of any vector field $X$ in $\mathfrak{g}$ is $-X$. This proves that any sub-Laplacian is a divergence form operator.

Furthermore, if Exp is the exponential map from $\mathfrak{g}$ to $\mathbb{G}$ and Log its inverse function, then (by making use of the triangular form of $\mathcal{J}_{\tau_{x}}(0)$ ) $\operatorname{Exp}$ and Log turn out to be globally defined diffeomorphisms with polynomial components and they commute with the dilations $\delta_{\lambda}$ (here we are assuming that $\mathfrak{g}$ is identified with $\mathbb{R}^{N}$ via the Jacobian coordinates).

Finally, the Jacobian determinant of Exp, Log, of the inversion and of right and left translations on $\mathbb{G}$ are identically one. In particular, the Lebesgue measure is a Haar measure on $\mathbb{G}$.

To end this survey of known facts, we recall a general result concerning with abstract Lie groups. Let $\varphi: \mathbb{G} \rightarrow \mathbb{H}$ be a Lie group morphism. Since $\varphi$ maps the identity of $\mathbb{G}$ into the identity of $\mathbb{H}$, then $\mathrm{d} \varphi$ (the differential of $\varphi$ ) is a linear map from the tangent space to $\mathbb{G}$ at the identity (say, $\mathbb{G}_{e}$ ) to the tangent space to $\mathbb{H}$ at the identity $\left(\right.$ say, $\mathbb{H}_{e}$ ). By means of the natural identification between $\mathbb{G}_{e}$ and $\mathfrak{g}$ (the Lie algebra of $\mathbb{G}$ ) and between $\mathbb{H}_{e}$ and $\mathfrak{h}$ (the Lie algebra of $\mathbb{H}), d \varphi$ induces a linear map from $\mathfrak{g}$ to $\mathfrak{h}$, which we shall still denote by $\mathrm{d} \varphi$. We have the following remarkable result (if $X \in \mathfrak{g}$ and $x \in \mathbb{G}$, we shall denote by $X_{x}$ the value of the vector field $X$ at the point $x$ ).

Theorem 5.2 (see e.g. [22, Theorem 3.14]) Let $\mathbb{G}$ and $\mathbb{H}$ be Lie groups with related Lie algebras $\mathfrak{g}$ and $\mathfrak{h}$, respectively. Let $\varphi: \mathbb{G} \rightarrow \mathbb{H}$ be a Lie group morphism. Then,

1. for every $X \in \mathfrak{g}$ and every $x \in \mathbb{G}$, we have $(\mathrm{d} \varphi(X))_{\varphi(x)}=\mathrm{d} \varphi\left(X_{x}\right)$;

2. $\mathrm{d} \varphi: \mathfrak{g} \rightarrow \mathfrak{h}$ is a Lie algebra morphism.

We now turn to recall some known properties of the exponential map. Let $\mathfrak{r}$ be the Lie algebra of $(\mathbb{R},+)$ and let $X \in \mathfrak{g}$ be fixed. The map $\psi: \mathfrak{r} \rightarrow \mathfrak{g}$, 
$\lambda \mathrm{d} / \mathrm{d} t \mapsto \lambda X$ is a Lie algebra morphism, whence there exists a unique Lie group morphism $\exp _{X}: \mathbb{R} \rightarrow \mathbb{G}$ whose differential is $\psi$. In particular, if $e$ denotes the identity of $\mathbb{G}$, we have

$$
\left.\frac{\mathrm{d}}{\mathrm{d} t}\right|_{t=0} f\left(\exp _{X}(t)\right)=X_{e}(f), \quad \forall f \in C^{\infty}(\mathbb{G}) .
$$

With the above notations, $\operatorname{Exp}: \mathfrak{g} \rightarrow \mathbb{G}$, defined by $\operatorname{Exp}(X):=\exp _{X}(1)$ is the exponential map related to $\mathbb{G}$. A useful property of Exp is the following one

$$
\operatorname{Exp}(t X)=\exp _{X}(t), \quad \forall t \in \mathbb{R}, X \in \mathfrak{g} .
$$

We are now able to prove Theorem 5.6, the main result of the Appendix. The definition of homogeneous Carnot group provided in Section 1 may seem slightly different from the one usually given in literature. Indeed (see e.g., $[8,18,21,13]$ ) a Carnot group (or stratified group) $\mathbb{H}$ is defined to be a connected and simply connected Lie group whose Lie algebra $\mathfrak{h}$ admits a stratification, i.e., a direct sum decomposition

$$
\mathfrak{h}=V_{1} \oplus V_{2} \oplus \cdots \oplus V_{r} \quad \text { such that } \quad\left\{\begin{array}{l}
{\left[V_{1}, V_{i-1}\right]=V_{i} \quad \text { if } \quad 2 \leq i \leq r} \\
{\left[V_{1}, V_{r}\right]=\{0\}}
\end{array}\right.
$$

Here, for any two subsets $V, W$ of $\mathfrak{h}$ we have let $[V, W]=\operatorname{span}\{[v, w] \mid v \in$ $V, w \in W\}$. As we prove in Proposition 5.3 and in Theorem 5.6 below, the operative definition of homogeneous Carnot group given in the introduction, is equivalent (up to isomorphism) to the classical one.

Proposition 5.3 A homogeneous Carnot group is a classical Carnot group.

Proof. Let $\mathbb{G}=\left(\mathbb{R}^{N}, \circ, \delta_{\lambda}\right)$ be a homogeneous Carnot group. Clearly $\mathbb{G}$ is connected and simply connected. For $i=1, \ldots, r$ and $j=1, \ldots, N_{i}$, let $Z_{j}^{(i)}$ be the vector field of $\mathfrak{g}$ agreeing with $\partial / \partial x_{j}^{(i)}$ at the origin. We set $V_{i}:=\operatorname{span}\left\{Z_{1}^{(i)}, \ldots, Z_{N_{i}}^{(i)}\right\}$. Then $\mathfrak{g}=V_{1} \oplus V_{2} \oplus \cdots \oplus V_{r}$. Moreover, we know that $Z_{j}^{(i)}$ is $\delta_{\lambda}$-homogeneous of degree $i$. This immediately yields $\left[V_{1}, V_{r}\right]=\{0\}$. Moreover, a field belonging to $\left[V_{1}, V_{i-1}\right]$ is $\delta_{\lambda}$-homogeneous of degree $i$, hence, as an element of $\mathfrak{g}=V_{1} \oplus \cdots \oplus V_{r}$, it must necessarily belong to $V_{i}$, by simple homogeneity arguments. This proves $\left[V_{1}, V_{i-1}\right] \subseteq V_{i}$.

On the other hand, by the definition of homogeneous Carnot group, any element $X$ of $V_{i}$ belongs to the Lie algebra generated by $Z_{1}^{(1)}, \ldots, Z_{N_{1}}^{(1)}$ : by homogeneity arguments, $X$ must necessarily be a linear combination of brackets of height $i$ of $Z_{1}^{(1)}, \ldots, Z_{N_{1}}^{(1)}$. In particular, it follows that $X$ belongs to $\left[V_{1}, V_{i-1}\right]$. Hence $V_{i} \subseteq\left[V_{1}, V_{i-1}\right]$ and the proposition is completely proved. 
Before proving that, up to isomorphism, the converse of Proposition 5.3 holds, we recall some results about the Campbell-Hausdorff formula. Let $\mathfrak{h}$ be a nilpotent Lie algebra. For $X, Y \in \mathfrak{h}$ we set $^{1}$

$$
\begin{aligned}
& X \diamond Y:=\sum_{n \geq 1} \frac{(-1)^{n+1}}{n} \sum_{\substack{p_{i}+q_{i} \geq 1 \\
1 \leq i \leq n}} \frac{(\operatorname{adX})^{p_{1}}(\operatorname{ad} \mathrm{Y})^{q_{1}} \cdots(\operatorname{adX})^{p_{n}}(\operatorname{ad} \mathrm{Y})^{q_{n}-1} Y}{\left(\sum_{j=1}^{n}\left(p_{j}+q_{j}\right)\right) p_{1} ! q_{1} ! \cdots p_{n} ! q_{n} !} \\
& =X+Y+\frac{1}{2}[X, Y]+\frac{1}{12}[X,[X, Y]]-\frac{1}{12}[Y,[X, Y]]+\text { brackets of height } \geq 4
\end{aligned}
$$

Since $\mathfrak{h}$ is nilpotent, (5.4) is a finite sum and $\diamond$ defines a composition law on $\mathfrak{h}$. The following remarkable result gives the well known Campbell-Hausdorff formula.

Theorem 5.4 (see e.g. [7, Theorem 1.2.1]) Let ( $\mathbb{H}, *$ ) be a connected and simply connected Lie group. Suppose that the Lie algebra $\mathfrak{h}$ of $\mathbb{H}$ is nilpotent. Then $\diamond$ defines a Lie group structure on $\mathfrak{h}$ and $\operatorname{Exp}:(\mathfrak{h}, \diamond) \rightarrow(\mathbb{H}, *)$ is a Lie group-isomorphism. In particular, we have

$$
\operatorname{Exp}(X) * \operatorname{Exp}(Y)=\operatorname{Exp}(X \diamond Y), \quad \forall X, Y \in \mathfrak{h} .
$$

If $\mathfrak{h}$ is a finite-dimensional Lie algebra, then (by the Third Fundamental Theorem of Lie, see [20, Theorem 3.15.1]) there exists a connected and simply connected Lie group whose Lie algebra is isomorphic to $\mathfrak{h}$. As a consequence, we obtain the following result.

Corollary 5.5 Let $\mathfrak{h}$ be a finite-dimensional nilpotent Lie algebra. Then $\diamond$ defines a Lie group structure on $\mathfrak{h}$. Moreover, if $\mathfrak{h}$ and $\mathfrak{g}$ are finitedimensional nilpotent Lie algebras and $\varphi: \mathfrak{h} \rightarrow \mathfrak{g}$ is a Lie algebra morphism, then $\varphi$ is also a Lie group morphism between $(\mathfrak{h}, \diamond)$ and $(\mathfrak{g}, \diamond)$.

The second assertion of the above remark holds since, for every $X, Y \in \mathfrak{h}$, $\varphi(X \diamond Y)$ equals

$$
\varphi(X)+\varphi(Y)+\frac{1}{2}[\varphi(X), \varphi(Y)]+\frac{1}{12}[\varphi(X),[\varphi(X), \varphi(Y)]]+\cdots=\varphi(X) \diamond \varphi(Y) .
$$

We are now in the position to prove the main result of the Appendix.

Theorem 5.6 Let $\mathbb{H}$ be a classical Carnot group. Then there exists a homogeneous Carnot group $\mathbb{G}$ isomorphic to $\mathbb{H}$. We can choose as $\mathbb{G}$ the Lie algebra of $\mathbb{H}$ (identified with $\mathbb{R}^{N}$ by a suitable choice of a basis) equipped with the composition law defined by the Campbell-Hausdorff formula. In this case, a group-isomorphism from $\mathbb{G}$ to $\mathbb{H}$ is the exponential map.

\footnotetext{
${ }^{1}$ We use the notation $(\operatorname{ad} A) B=[A, B]$. Moreover, if $q_{n}=0$, the term in the sum (5.4) is by convention $\cdots(\operatorname{adX})^{p_{n-1}}(\operatorname{ad~} \mathrm{Y})^{p_{n-1}}(\operatorname{adX})^{p_{n}-1} X$. Clearly, if $q_{n}>1$, or $q_{n}=0$ and $p_{n}>1$, the term is zero.
} 
Proof. Let $(\mathbb{H}, *)$ be a Carnot group, according to the classical definition. Let $\mathfrak{h}$ be the algebra of $\mathbb{H}$. Let $\mathfrak{h}=V_{1} \oplus \cdots \oplus V_{r}$ be a stratification as in (5.3). Obviously, the brackets in $\mathfrak{h}$ of height $>r$ vanish. Thus $\mathfrak{h}$ is nilpotent. Then, Theorem 5.4 yields that $\operatorname{Exp}:(\mathfrak{h}, \diamond) \rightarrow(\mathbb{H}, *)$ is a Lie group-isomorphism when $\diamond$ is as in (5.4). We now prove that $(\mathfrak{h}, \diamond)$ is a homogeneous Carnot group according to the definition in Section 1.

We fix a basis for $\mathfrak{h}$ adapted to its stratification: for $i=1, \ldots, r$, set $N_{i}:=\operatorname{dim} V_{i}$ and let $\left(E_{1}^{(i)}, \ldots, E_{N_{i}}^{(i)}\right)$ be a basis for $V_{i}$. Then consider the basis for $\mathfrak{h}$ given by

$$
E_{1}^{(1)}, \ldots, E_{N_{1}}^{(1)} ; \ldots ; E_{1}^{(r)}, \ldots, E_{N_{r}}^{(r)} .
$$

By means of this basis, we fix a coordinate system on $\mathfrak{h}$ and we identify $\mathfrak{h}$ with $\mathbb{R}^{N}$, where $N:=N_{1}+\cdots+N_{r}$. We then define a family of dilations $\left\{\delta_{\lambda}\right\}_{\lambda>0}$ as follows:

$$
\delta_{\lambda}: \mathfrak{h} \rightarrow \mathfrak{h}, \quad \delta_{\lambda}\left(\sum_{i=1}^{r} X_{i}\right):=\sum_{i=1}^{r} \lambda^{i} X_{i}, \quad \text { where } X_{i} \in V_{i}
$$

With the above choice of coordinates, $\delta_{\lambda}$ is as in (1.1). We next show that $\delta_{\lambda}$ is an automorphism of $(\mathfrak{h}, \diamond)$. Recalling Remark 5.5 , it is enough to prove

$$
\delta_{\lambda}([X, Y])=\left[\delta_{\lambda}(X), \delta_{\lambda}(Y)\right],
$$

for every $X, Y \in \mathfrak{h}$. If $X=\sum_{i=1}^{r} X_{i}$ and $Y=\sum_{i=1}^{r} Y_{i}$, where $X_{i}, Y_{i} \in V_{i}$, we have $\left[X_{i}, Y_{j}\right] \in V_{i+j}$, whence $\delta_{\lambda}([X, Y])$ equals

$$
\begin{aligned}
\sum_{i, j=1}^{r} \delta_{\lambda}\left(\left[X_{i}, Y_{j}\right]\right) & =\sum_{i, j=1}^{r} \lambda^{i+j}\left[X_{i}, Y_{j}\right] \\
& =\sum_{i, j=1}^{r}\left[\delta_{\lambda}\left(X_{i}\right), \delta_{\lambda}\left(Y_{j}\right)\right]=\left[\delta_{\lambda}(X), \delta_{\lambda}(Y)\right] .
\end{aligned}
$$

Let now $\mathfrak{g}$ be the Lie algebra of $(\mathfrak{h}, \diamond)$. The proof is complete if we show that the Lie algebra generated by the $N_{1}$ vector fields $Z_{1}, \ldots, Z_{N_{1}}$ in $\mathfrak{g}$ agreeing at the origin with the first $N_{1}$ partial derivatives coincides with the whole $\mathfrak{g}$. Since Exp $: \mathfrak{h} \rightarrow \mathbb{H}$ is a Lie group-isomorphism, then $d \operatorname{Exp}: \mathfrak{g} \rightarrow \mathfrak{h}$ is a Lie algebra-isomorphism (see Theorem 5.2). Since moreover $E_{1}^{(1)}, \ldots, E_{N_{1}}^{(1)}$ is a system of Lie-generators for $\mathfrak{h}$, it is enough to prove that for all $k=1, \ldots, N_{1}$ we have $\mathrm{d} \operatorname{Exp}\left(Z_{k}\right)=E_{k}^{(1)}$. To show this, being a left-invariant vector field determined by its value at the identity, it suffices to prove

$$
\left(\mathrm{d} \operatorname{Exp}\left(Z_{k}\right)\right)_{e}=\left(E_{k}^{(1)}\right)_{e} .
$$


For every $f \in C^{\infty}(\mathbb{H})$, we have (see (5.1) and (5.2))

$$
\begin{aligned}
\left(\mathrm{d} \operatorname{Exp}\left(Z_{k}\right)\right)_{e}(f) & =\left(\mathrm{d} \operatorname{Exp}\left(Z_{k}\right)_{0}\right)(f)=\left(Z_{k}\right)_{0}(f \circ \operatorname{Exp}) \\
& =\left.\left(\partial / \partial x_{k}^{(1)}\right)\right|_{x=0} f\left(\operatorname{Exp}\left(\sum_{i=1}^{r} \sum_{j=1}^{N_{i}} x_{j}^{(i)} E_{j}^{(i)}\right)\right) \\
& =\left.\frac{\mathrm{d}}{\mathrm{d} t}\right|_{t=0} f\left(\operatorname{Exp}\left(t E_{k}^{(1)}\right)\right)=\left.\frac{\mathrm{d}}{\mathrm{d} t}\right|_{t=0} f\left(\exp _{E_{k}^{(1)}}(t)\right)=\left(E_{k}^{(1)}\right)_{e}(f) .
\end{aligned}
$$

The theorem is thus completely proved.

From Theorem 5.6 it is evident that any Carnot group is isomorphic to a homogeneous Carnot group for which the group inverse is simply given by $x^{-1}=-x$.

\section{References}

[1] Beals, R., Gaveau, B., Greiner, P.: The Green function of model step two hypoelliptic operators and the analysis of certain tangential Cauchy Riemann complexes. Adv. Math. 121 (1996), no. 2, 288-345.

[2] Bonfiglioli, A., Lanconelli, E., Uguzzoni, F.: Uniform Gaussian estimates of the fundamental solutions for heat operators on Carnot groups. Adv. Differential Equations 7 (2002), 1153-1192.

[3] Bonfiglioli, A., Lanconelli, E., Uguzzoni, F.: Fundamental solutions for non-divergence form operators on stratified groups. Trans. Amer. Math. Soc. 356 (2004), no. 7, 2709-2737.

[4] Bonfiglioli, A., Uguzzoni, F.: Families of diffeomorphic sub-Laplacians and free Carnot groups. Forum Math. 16 (2004), no. 3, 403-415.

[5] Citti, G., Lanconelli, E., Montanari, A.: Smoothness of Lipschitz continuous graphs with nonvanishing Levi curvature. Acta Math. 188 (2002), 87-128.

[6] Citti, G., Montanari, A.: Strong solutions for the Levi curvature equation, Adv. Differential Equations 5 (2000), 323-342.

[7] Corwin, L. J., Greenleaf, F.P.: Representations of nilpotent Lie groups and their applications (Part I: Basic theory and examples). Cambridge Studies in Advanced Mathematics 18. Cambridge University Press, Cambridge, 1990.

[8] Folland, G. B.: Subelliptic estimates and function spaces on nilpotent Lie groups. Ark. Mat. 13 (1975), 161-207.

[9] Folland, G. B.: On the Rothschild-Stein lifting theorem. Comm. Partial Differential Equations 2 (1977), 165-191.

[10] Folland, G. B., Stein, E. M.: Hardy spaces on homogeneous groups. Mathematical Notes 28. Princeton University Press, Princeton, N.J., 1982.

[11] Goodman, R. W.: Lifting vector fields to nilpotent Lie groups. J. Math. Pures Appl. 57 (1978), 77-85. 
[12] Grayson, M., Grossman, R.: Models for free nilpotent Lie algebras. J. Algebra 135 (1990), 177-191.

[13] HajŁasz, P., Koskela, P.: Sobolev met Poincaré. Mem. Amer. Math. Soc. 145 (2000), no. 688.

[14] Hall, M.: A basis for free Lie rings and higher commutators in free groups. Proc. Amer. Math. Soc. 1 (1950), 575-581.

[15] Hörmander, L.: Hypoelliptic second order differential equations. Acta Math. 119 (1967), 147-171.

[16] Hörmander, L., Melin, A.: Free systems of vector fields. Ark. Mat. 16 (1978), no. 1, 83-88.

[17] Huisken, G., Klingenberg, W.: Flow of real hypersurfaces by the trace of the Levi form. Math. Res. Lett. 6 (1999), 645-661.

[18] Rothschild, L.P., Stein, E.M.: Hypoelliptic differential operators and nilpotent groups. Acta Math. 137 (1976), 247-320.

[19] Slodkowski, Z., Tomassini, G.: Weak solutions for the Levi equation and envelope of holomorphy. J. Funct. Anal. 101 (1991), 392-407.

[20] Varadarajan, V. S.: Lie groups, Lie algebras and their representations, Graduate Texts in Mathematics 102. Springer-Verlag, New York, 1984.

[21] Varopoulos, N. T., Saloff-Coste, L., Coulhon, T.: Analysis and geometry on groups. Cambridge Tracts in Mathematics 100. Cambridge University Press, Cambridge, 1992.

[22] Warner, F.W.: Foundations of differentiable manifolds and Lie groups. Graduate Texts in Mathematics 94. Springer-Verlag, New York-Berlin, 1983.

Recibido: 10 de septiembre de 2003

Andrea Bonfiglioli

Dipartimento di Matematica Università degli Studi di Bologna

Piazza di Porta S. Donato, 5 40126 Bologna, Italy. bonfigli@dm. unibo.it

Francesco Uguzzoni

Dipartimento di Matematica Università degli Studi di Bologna

Piazza di Porta S. Donato, 5 40126 Bologna, Italy. uguzzoni@dm. unibo.it

\footnotetext{
Investigation supported by University of Bologna. Funds for selected research topics.
} 